layered structures based on these semimagnetic semiconductors is focused primarily on two families of systems. The first is multilayers and heterostructures derived from the II-VI semiconducting materials $^{3}$ (such as CdTe and $\mathrm{ZnSe}$ ), in which the magnetic constituents are isostructural Mn compounds (MnTe, $\mathrm{MnSe}$ ). The other is based on IV-VI semiconductors such as $\mathrm{PbTe}$ and rareearth magnetic chalcogenides ${ }^{4}$ such as EuTe. Some of the unusual magnetic properties of these systems are of great fundamental interest, for instance the formation of 'spin superlattices' in which the electronic spin-up and spin-down states become spatially separated ${ }^{5,6}$. Also, results $^{7}$ from digital heterostructures (quantum wells in which the electronic states are tailored through controlled distribution of two-dimensional magnetic layers) confirm that multilayered magnetic semiconductor structures offer considerable technological opportunities: the Faraday effects are two orders of magnitude stronger than in bulk semimagnetic semiconductors, and the dynamics of the spin-dependent electronic processes are extraordinarily fast, and should thus be capable of handling much faster signal transmissions than in current state-of-theart communications.

As yet, though, technologists develop- ing new magnetic semiconductor quantum-well structures have been struggling to overcome two challenges. The first is fabrication of magnetic semiconductor structures with ferromagnetic components. By a caprice of nature, magnetic compounds that can be combined with the most widely used semiconducting materials are almost exclusively antiferromagnets, with no net magnetic moment. Systems with ferromagnetic components would be far more sensitive to external magnetic fields, further enhancing their technological value. The second challenge is to grow magnetic semiconducting structures based on the III-V compounds (such as GaAs, InSb) which are particularly advantageous in optoelectronic applications.

Set against this background, we can see that the work of Shi et al. represents a great step towards overcoming both these obstacles. The host material used in their work, GaAs, is representative of the III-V family; and the micrograins implanted are ferromagnetic. Furthermore, the Curie temperature for this GaMn modification is well above room temperature.

Of course, problems remain. At present, the new technique produces a random distribution of GaMn grains. It seems unlikely that it can be used for preparing continuous layers, as in systems fabricated by molecular beam epitaxy. But the recent successes of focused ion beam techniques ${ }^{8}$ suggest that the preparation of patterned ferromagnetic arrays is possible. It should be noted that the newest trend in research on magnetic semiconductor multilayers is to study systems with modulated structures within the layers for example, regular patterns of magnetic dots or 'wires' buried in the material. Once more control is gained over the process of GaMn precipitation, the method described by Shi et al. may become an ideal tool for fabricating such specimens.

Tom M. Giebultowicz is in the Department of Physics, Oregon State University, Corvallis, Oregon 97331, USA. Valerie Nunez is in the Reactor Radiation Division, National Institute of Standards and Technology, Gaithersburg, Maryland 20899, USA.

1. Shi, J. et at Nature 377, 707-710 (1995).

2. Luo, H. \& Furdyna, J. K. Semiconductor Science and Technology 10, 1041 (1995)

3. Diluted Magnetic Semiconductors (Semiconductors and Semimetals, vol. 25) (eds Furdyna, J. K. \& Kossut, J.) (Academic, Boston, 1988).

4. Yuan, S., Springholz, G., Bauer, G. \& Kriechbaum, M. Phys. Rev. B49, 5476 (1994).

5. Chou, W. C., Petrou, A., Warnock, J. \& Jonker, B. T. Phys Rev. Lett. 67, 3820 (1991).

6. Dai, N. et al. Phys. Rev. Lett. 67, 3824 (1991).

7. Crooker, S. A. et al. Phys. Rev. Lett. 75, 505 (1995).

Kikkawa, J. M. et al. Phys. Rev. B50, 2003-2006 (1994).

\title{
Don't hold your breath
}

EXIINCTION may already have claimed an otherwise revolutionary new form of vertebrate life: an erstwhile land animal with relatively huge, gaping jaws (pictured here), that lacks limbs, lungs, pulmonary circulation and even internal nostrils. The species concerned is a caecilian, Typhlonectes eiselti, and according to Ronald A. Nussbaum and Mark Wilkinson (Proc. $R$. Soc. Lond. B 261, 331-335; 1995), its unusual features could mark the start of an adaptive radiation of secondarily aquatic vertebrates.

Caecilians constitute the least known of the three living orders of amphibian. All extant species are limbless, although this condition is secondary (see Nature 365, 246-250; 1993). Their tropical distribution and mainly burrowing or aquatic lifestyle has ensured their obscurity: many of the 160 or so species recognized are known from just one or two specimens. Typhlonectes eiselti is known only from a single preserved specimen in the Vienna Museum of Natural History, collected somewhere in South America, no later than the 1920 s, and possibly much earlier.

Amphibians ventilate by pumping air into their lungs by way of the mouth cavity and internal nostrils, or choanae.
The fact that the choanae in $T$. eiselti are sealed, a condition unique in tetrapods, caught Nussbaum and Wilkinson's attention, and dissection revealed a complete lack of lungs and supporting pulmonary circulation. Freedom from the necessity of buccal venti-

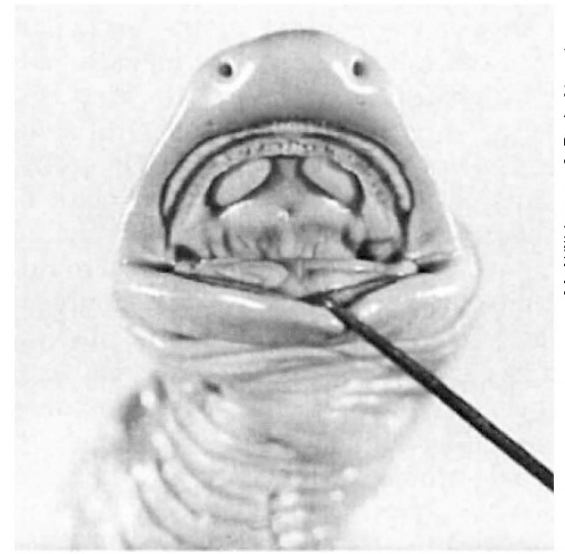

lation has allowed an extraordinary redesign of the skull. It is larger and looser than most caecilian skulls, with distinctly aberrant musculature, and a jaw articulation placed well back that allows for a very wide gape. This complex of features - the obligate aquatic lifestyle and redesigned skull - has the potential to free $T$. eiselti from the usual caecilian burrowing habit, and permit the foundation of an entirely new type of aquatic vertebrate (one thinks of Megachasma, the recently described genus of deep-sea shark).

What of lunglessness? Many amphibians use the skin as a gas-exchange surface, and some aquatic salamanders either retain lungs as buoyancy aids, or dispense with them altogether. Lunglessness tends to be associated with small size, low temperatures (and thus low metabolic rate) and high ambient oxygen concentration, such as one might find in fast-flowing mountain streams. Typh/onectes eiselti is 725 $\mathrm{mm}$ long, and is by far the largest lungless vertebrate known, something that would limit its environmental choices. Although the details of where it was collected are unknown, Nussbaum and Wilkinson suggest that it might inhabit cool, fast-moving streams in montane rainforests.

The fact that such habitats are currently threatened by human activity and that no other specimens of this species have been collected for at least 70 years - suggests that this bold experiment in vertebrate design might have met an untimely end.

H. G. 